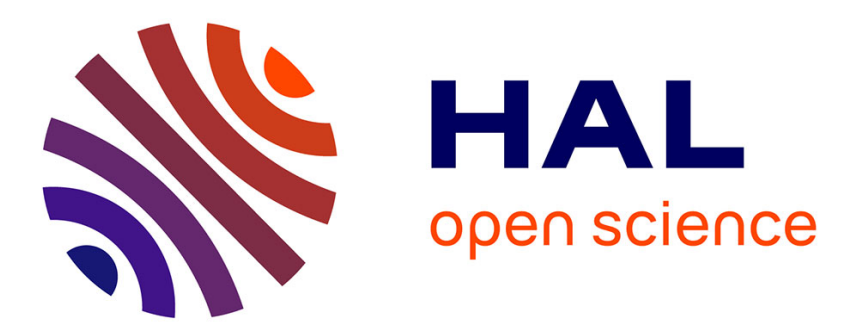

\title{
Urbanisme et transport dans les régions urbaines : enjeux et perspectives d'un urbanisme orienté vers le rail
}

Alain L'Hostis, Gebhard Wulfhorst, Benjamin Puccio, Catherine

Araud-Ruyant

\section{- To cite this version:}

Alain L'Hostis, Gebhard Wulfhorst, Benjamin Puccio, Catherine Araud-Ruyant. Urbanisme et transport dans les régions urbaines: enjeux et perspectives d'un urbanisme orienté vers le rail. RTS Recherche Transports Sécurité, 2007, 94, pp.11-26. 10.3166/rts.94.11-26 . hal-00145400

\section{HAL Id: hal-00145400 \\ https://hal.science/hal-00145400}

Submitted on 10 May 2007

HAL is a multi-disciplinary open access archive for the deposit and dissemination of scientific research documents, whether they are published or not. The documents may come from teaching and research institutions in France or abroad, or from public or private research centers.
L'archive ouverte pluridisciplinaire HAL, est destinée au dépôt et à la diffusion de documents scientifiques de niveau recherche, publiés ou non, émanant des établissements d'enseignement et de recherche français ou étrangers, des laboratoires publics ou privés. 
RECHERCHE TRANSPORTS SECURITE nº 94 JANVIER-MARS 2007

\title{
Urbanisme et transport dans les régions urbaines Enjeux et perspectives d'un urbanisme orienté vers le rail
}

\section{Urban planning and transport in urban regions Issues and outlook for rail-oriented urban development}

\author{
Gebhard Wulfhorst a, Alain L’Hostis b*, Benjamin Puccio c \\ Adresses des auteurs à la date d'acceptation de l'article \\ a Technische Universität München, Arcisstraße 21, 80333 München, Allemagne \\ b INRET-LVMT, 20 rue Élisée Reclus, BP 317, 59666 Villeneuve-d’Ascq cedex \\ c ADEUS Agence d’Urbanisme de Strasbourg, 9 rue Brûlée, BP 47R2, 67002 Strasbourg
}

Article reçu le 4 juillet 2006 ; accepté le 13 mars 2007

courriel : alain.lhostis@inrets.fr

\begin{abstract}
Résumé L’objet de cet article est d'exposer une approche du couple associant ville et transport ferroviaire. Le questionnement porte sur l'analyse des potentialités de développement des réseaux de transport ferroviaire, celle des potentialités de développement urbain, ainsi que celle de leurs interactions. Pour appréhender le couple associant transport ferroviaire et développement urbain, les formes de la coordination sont analysées en termes de secteurs d'intervention, de spatialité, de temporalité, ainsi qu'en termes organisationnels et méthodologiques. En prenant appui sur ces principes généraux, il a été possible d'étudier, dans le cadre du projet Bahn.Ville 1, des expériences d'urbanisme orienté vers le rail menées en Allemagne et en France. Les enseignements de ce projet doivent maintenant faire l'objet d'une mise en œuvre sur le terrain. La ligne ferroviaire Saint-Étienne-Firminy qui possède de fortes potentialités de développement et de renouvellement urbain en lien avec le mode ferré a été choisie à cet effet.
\end{abstract}

\begin{abstract}
This paper presents an analysis of the pair formed by the association between a city and rail transport. It will examine the potential for the development of rail networks, the potential for urban development and the interactions between the two. In order to analyze the pair formed by rail transport and urban development, the types of coordination have been analyzed, with regards to sector of intervention and spatial, temporal, organizational and methodological aspects. Based on these general principles, the Bahn.Ville project has investigated experience of rail-oriented urban development in both Germany and France. It now remains to implement the lessons learnt from this project in the field, which is to be done on the Saint-Étienne-Firminy line, where the potential for rail-based development and renewal is considerable.
\end{abstract}

Mots-clés Urbanisme ; Transport ferroviaire ; Développement urbain ; Région urbaine ; Coordination transport-urbanisme

Keywords Urban planning; Rail transport; Urban development; Urban region; Coordination between transport and urban planning 


\section{Introduction}

L'objet de cet article est d'exposer une approche du couple associant ville et transport ferroviaire. Le questionnement porte sur l'analyse des potentialités de développement des réseaux de transport ferroviaire, celle des potentialités de développement urbain, ainsi que celle de leurs interactions. Pour analyser les relations entre le mode ferroviaire et la ville, l'échelle pertinente est celle de la région urbaine qui intègre l'agglomération à un espace élargi tant du point de vue fonctionnel qu'institutionnel. Les méthodes mobilisées relèvent de l'analyse territoriale, avec l'étude de la mobilité et des préférences des individus, l'analyse de l'offre de transport public centrée sur le train, l'étude des dynamiques du développement urbain, ainsi que celle du cadre institutionnel et des procédures en jeu dans la coordination des politiques urbaines et des politiques de transport.

Pour traiter ce questionnement le texte est organisé en quatre parties. Nous présentons les principes généraux de l'organisation urbaine et des orientations possibles pour les politiques de développement urbain. Ensuite nous proposons un cadre d'analyse pour envisager le couple associant ville et transport et pour identifier des leviers d'action pertinents. La troisième partie expose les résultats marquants du projet de recherche Bahn.Ville 1 qui visait à analyser une série d'expériences en France et en Allemagne portant sur des développements urbains orientés vers le rail. Les éléments présentés dans l'article proviennent pour l'essentiel de quatre terrains, situés en France et en Allemagne complétée par des éclairages sur le cas de Karlsruhe ; il s'agit de lignes ferroviaires inscrites dans les agglomérations de Strasbourg, Nantes, Ravensburg et Bonn. Enfin la dernière section présente les premiers éléments d'orientation d'un projet de recherche à venir ayant pour objet la mise en œuvre des éléments recueillis dans le projet Bahn.Ville sur deux terrains, l’un situé en France, dans la région de Saint-Étienne, et l'autre en Allemagne dans l'agglomération de Bonn.

\section{Les principes d'une organisation urbaine en phase avec les réseaux de transport ferroviaire}

Les réflexions sur l'avenir de la ville européenne mettent en avant des formes de plus en plus polycentriques et multipolaires. Ainsi, Peter Hall (1999) propose-t-il une relecture à l'aune de la métropolisation du modèle de Christaller, dans lequel la ville européenne, façonnée par l'évolution du système des mobilités, apparaît comme fortement structurée autour des centres anciens et adossée à de nouvelles polarités. Ce développement de la ville, bien que largement conditionné par celui de l'automobile (Wiel, 1999), (Mangin, 2004), est générateur de centralités au sein desquelles et entre lesquelles les transports publics, et en particulier le mode ferroviaire, peuvent jouer un rôle de structuration important.

Le fait métropolitain, compris comme une structuration spatiale à partir d'objets urbains qui rayonnent à l'échelle globale, passe par la réunion de fonctions supérieures. La volonté de positionnement des territoires à l'intérieur de l'Europe fait émerger bon nombre d'activités orientées vers le rayonnement des métropoles. La notion de métropole implique que le pôle central fonctionne comme un moteur pour d'autres pôles, notamment pour les fonctions de décision et contrôle, d'innovation et de compétition, d'accessibilité et d'échange. Cette idée implique donc une organisation en réseau de plusieurs pôles, fonctionnant sur un mode hiérarchique à l'intérieur de la région urbaine. Dans les contextes transfrontaliers, les différentiels en matière d'économie, de langues, de cultures créent souvent des tensions, concurrences et conflits supplémentaires. Pour tirer parti de ces différences, on cherche à mettre en valeur les complémentarités et synergies au profit d'une dimension européenne d'une part, 
d'une qualité de vie locale d'autre part. Il s’agit alors, en termes d'intégration spatiale, de renforcer les échelles régionales stratégiques, sans pour autant nier les identités locales.

Si le polycentrisme est vu par certains auteurs comme une structure émergente dans l'espace urbain (Dubois-Taine, 2001), (Aguiléra et Mignot, 2002), (Bertolini \& Dijst, 2003), (Parr, 2004), cette figure de la ville organisée autour de plusieurs centres correspond aussi à un des modèles d'organisation de l'aménagement à toutes les échelles (Faludi \& Waterhout, 2002), (Parr, 2004), (Baudelle et Peyrony, 2005), (Sykes, 2005). De l'aménagement du territoire de l’Union européenne (CCE, 1999) à l'organisation des métropoles et des régions urbaines (Parr, 2004), sans oublier les orientations nationales (DATAR, 2000), toutes les orientations assumées des politiques spatiales accordent une place centrale au concept du polycentrisme.

Qu'on la voie comme un processus à l'œuvre ou bien comme un futur souhaitable, la figure polycentrique ne s’inscrit pas dans une volonté de réduire les mobilités (Aguiléra et Mignot, 2002). Au contraire, c'est l'organisation, la canalisation des flux dans la ville qui doit venir appuyer cette option spatiale. Dans ce contexte, réintroduire le rail dans la ville ou susciter une forme urbaine favorable au mode ferré constitue un champ d’opportunité pour la ville européenne (Dupuy, 1991), (Troin, 1995), (Menerault et al., 2006a). D’un point de vue normatif, ces formes d'action peuvent aussi être vues comme les déclinaisons des principes d'un développement urbain durable (Rouxel et Rist, 2000), orienté vers la construction d'une alternative à l'automobilité.

Les réseaux et les services de transports font partie des éléments pris en compte par les acteurs privés et publics dans les choix de localisation des ménages, des entreprises et des équipements. Si l'on souhaite aujourd'hui orienter la structure territoriale par les réseaux des transports en commun, il est nécessaire de mettre l'accent sur une bonne accessibilité des pôles du territoire et de considérer le réseau ferroviaire comme réseau prioritaire au niveau des régions urbaines. Entre les polarités de la ville émergente, le fer doit être pensé comme inscrit dans un réseau de transport collectif à l'échelle de la région urbaine tout autant que connecté avec l’extérieur.

Si les réseaux jouent un rôle essentiel, l'organisation des territoires urbains n'est absolument pas neutre dans la génération des flux de déplacement. Densité urbaine, mixité et polycentrisme peuvent être vus comme trois modèles fondamentaux pour l'organisation de la ville (Vodoz, 2004). Mais une politique globale à l'échelle de la région urbaine peut chercher à combiner ces trois modes d'intervention avec :

- la densité pour contrer la tendance à l’étalement urbain imputable à l’automobilité ;

- la mixité pour contrer l’urbanisme aréolaire et ses espaces monofonctionnels ;

- le polycentrisme comme alternative au modèle centre-périphérie.

La densité urbaine et la mixité fonctionnelle et sociale assurent l'économie d'espace et la diversité du système urbain. Des contacts de proximité et la réduction des distances parcourues sont rendus possible, mais ils ne sont pas garantis. Compte tenu de la mobilité quotidienne, une armature urbaine hiérarchique bien desservie en transport collectif permet de garder des options de choix qui ne dépendent pas exclusivement de l'automobile.

L’évolution actuelle des villes peut ménager des opportunités pour le développement des réseaux de transport en commun. Cependant, pour saisir ces opportunités, il est nécessaire de considérer les interrelations étroites entre la ville et les transports. 


\section{La ville et les transports comme système}

De nombreux auteurs ont monté que, si la ville produit ses propres réseaux de transport, elle est aussi largement façonnée par ceux-ci (Dupuy 1991), (Ragon 1995), (Beaucire, 1996), (Bretagnolle, 1999), (Menerault et Barré, 2001), (Richards, 2001), (Wachter, 2004), (Menerault et al., 2006b). Ville et transport construisent une relation dialectique qui doit être parcourue dans les deux sens. Si le développement des réseaux de transport doit être conçu comme un outil au service d'un projet de ville ou de territoire, envisager l'autre sens de la relation incite à penser une ville qui tire parti des contraintes et des opportunités des réseaux de transport.

Le réseau de relations dynamiques liant la ville et les transports peut être schématisée à partir du diagramme de la Fig. 1. Le développement urbain et l'organisation des transports sont ici vus comme des processus de long terme tandis que la mobilité quotidienne s'inscrit dans un processus de court terme. Les mobilités sont structurées par les activités et les structures territoriales et forment la demande de transport. En particulier, la mobilité quotidienne se réalise à partir des structures spatiales afin de lier les différents lieux d'activités. Du côté des processus longs, la structure urbaine et le système de transport sont liés au travers de l'accessibilité. Dans ce cadre, ce sont des interactions et des processus dynamiques qui conditionnent le développement futur des structures urbaines.

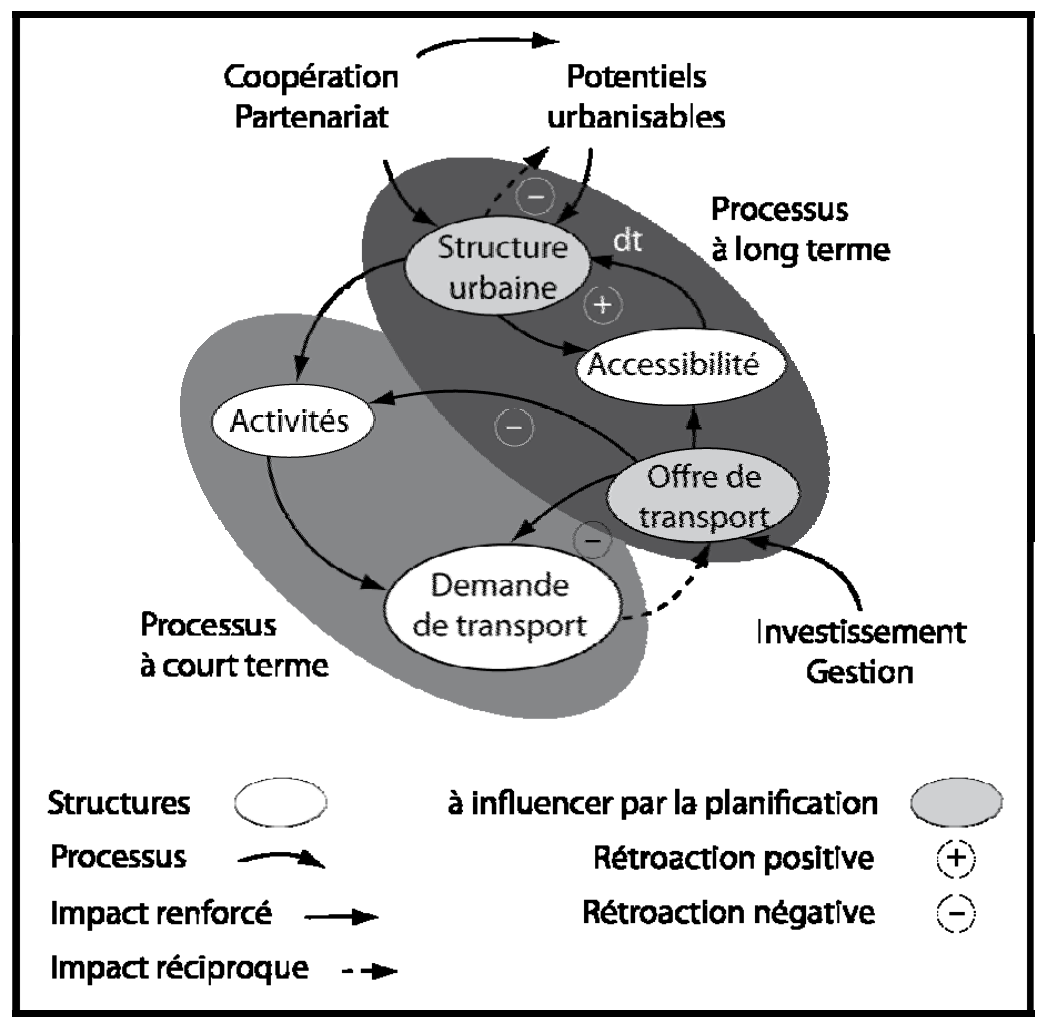

Fig. 1 Schéma des interactions dynamiques entre urbanisme et transport D’après (Wulfhorst, 2003)

Dans la structuration des politiques publiques, les transports font l'objet, à côté du logement, du développement économique ou du foncier, d'une des politiques sectorielles qui interfère avec les champs de l'urbanisme et de l'aménagement. 
Les analyses de la relation entre forme urbaine et transport admettent l'idée que l'articulation entre les politiques de transport et les politiques d'aménagement est une clé d'un développement urbain durable (Bertolini et al., 2005), devant l'amélioration des transports publics ou la réduction de la place et de l'usage de la voiture (Laconte, 1999). Pour Marc Wiel, c’est du décloisonnement des logiques sectorielles et des intérêts territoriaux aux échelons locaux que peut venir la réponse aux défis du séisme de la mobilité et de la transformation de la ville (Wiel, 1999).

Si l'identification des principaux leviers fait partie d'un diagnostic largement partagé, les analyses des expériences des villes d'Europe ${ }^{1}$ mettent en avant les difficultés de mise en œuvre de la coordination des politiques de transport et d'urbanisme : questions institutionnelles, incohérences dans la définition des objectifs, télescopage des échelles spatiales (Offner, 1997), (Jouve, 2003),(Kaufmann et al., 2003), (Schwanen et al., 2004), (Pretsch et al., 2005). En effet, le développement de projets urbains s'appuyant sur le mode ferroviaire suppose la mobilisation et la coordination d'un large ensemble d'acteurs ; le partenariat doit jouer un rôle essentiel (Klaer Blanchard, 1999) (Puccio et Wulfhorst, 2005).

Pour envisager les différentes dimensions de la coordination des politiques de transport et d'urbanisme d'un point de vue théorique Wulfhorst (2003 et 2006) propose une grille de lecture globale. Le tableau décline les cinq dimensions de la coordination.

\section{Tableau Les dimensions de la coordination entre urbanisme et transport dans les régions urbaines}

D’après (Wulfhorst, 2006)

Les développements suivants, déclinant les cinq dimensions de la coordination, sont issus d'analyses menées dans différentes régions en Europe et aux États-Unis dans le cadre d'un projet de recherche du programme Marie-Curie intitulé TRANSFORMES (intégration entre transports et forme urbaine dans la région européenne de Strasbourg) (Wulfhorst, 2006).

\subsection{La coordination transversale}

Une coordination transversale entre les différents modes de transports, entre transports et aménagement et avec les autres domaines économiques, écologiques et sociaux renvoie à la question de la mise en cohérence des différentes stratégies et actions des acteurs publics et privés.

La coordination transversale concerne aussi une politique des transports assemblant les différents services de transports en commun dans un système performant (par exemple interconnexion entre desserte TGV, services régionaux et transport collectif urbain; gestion commune des différentes autorités organisatrices des transports au niveau régional) afin d'assurer l'arbitrage entre les différents modes de déplacement, notamment entre la voiture particulière et les transports en commun, mais aussi envers la marche à pied et le vélo.

\subsection{La coordination spatiale}

\footnotetext{
${ }^{1}$ L’attention portée à la ville européenne ne doit toutefois pas faire oublier des expériences autres, avec en particulier le Transit Oriented Development développé aux États-Unis dans le cadre du New Urbanism (Mangin, 2004), (Cervero \& Kochelman, 1997), (CDT, 2002).
} 
Le fait urbain se caractérise par la dilatation des espaces et par l'articulation croissante entre les différents niveaux. Ainsi, la métropole organise sa région urbaine d'une part et participe aux échanges à l'échelle globale d'autre part. C'est dans l'articulation et la coordination des échelles qu'il faut envisager la coordination entre urbanisme et transport.

Dans un système de mobilité ouvert, ne se limitant pas à un territoire découpé par les compétences politiques et circonscriptions administratives, les territoires fonctionnels sont presque systématiquement en discordance avec les périmètres institutionnels, les espaces d'activités et les flux d'échanges.

D’un côté il y a donc à mener une recherche de cohérence entre les zones d'influence fonctionnelles (les aires urbaines par exemple) et les autorités publiques compétentes de ces territoires (par exemple АOT). D’un autre côté il y aura toujours un besoin de coopération entre entités voisines (communes) et entre les différents niveaux spatiaux (commune, département, région, État...).

On observe des imbrications d'échelles, car d'une part des installations locales induisent souvent des impacts au niveau régional, d'autre part une problématique globale nécessite des actions concrètes au niveau local. Les interactions et conflits ont lieu sur différents niveaux d'échelles spatiales. Certains conflits locaux sont à prendre en compte, à traiter, à résoudre à un niveau spatial supérieur. Ainsi, un morcellement des compétences en matière d'aménagement du territoire sur un même territoire est à éviter ; les principes de subsidiarité doivent alors être favorisés.

Aujourd'hui, l'échelle de la région urbaine prend de plus en plus de poids dans les interactions fonctionnelles des territoires (Parr, 2004), mais la compétence politique et institutionnelle reste encore largement à renforcer.

Les activités, la mobilité quotidienne et les choix de localisation à long terme s’organisent à l'échelle des régions urbaines. Par contre, on constate un manque d'acteur, un manque d'autorité, de responsabilité face aux problèmes rencontrés à ce niveau territorial : l'intermodalité des transports et l'étalement urbain sont deux exemples de ces problématiques qui transgressent les territoires.

\subsection{La coordination temporelle}

Les temps de la ville incluent les temps quotidiens des mouvements et des flux, les temps des mobilités résidentielles, ainsi que les temps des projets et des régulations politiques. Dans le domaine des transports aussi, le temps court de l'évolution de la demande doit composer avec le temps long de la planification des réseaux.

En matière de transport comme d'urbanisme, les décisions d'aujourd'hui montreront leurs effets seulement dans le futur. Il est donc nécessaire d'anticiper en estimant les impacts des mesures dans un contexte temporel dynamique. Remarquons en outre que souvent les décisions ne peuvent être prises que dans un contexte particulier du fait de contraintes liées aux influences externes (événements, élections, crises), donc dans une fenêtre temporelle limitée. Si elles ne sont pas prises à un moment donné, elles ne seront jamais prises — ou du moins pas de la même manière.

Dans ce contexte, une vision à long terme est nécessaire pour orienter les processus, les séquences à moyen terme (stratégies) et les mesures à court terme (actions). Les démarches de planifications d'actions peuvent servir de référence. 
La coordination temporelle passe donc par :

- une vision du futur posée comme objectif (en allemand Leitbild) ; il faut savoir clairement où l'on veut arriver pour prendre les bonnes décisions en cours de chemin ; cette vision devrait être élaborée par tous les acteurs conjointement, et partagée au niveau politique, de sorte qu'elle puisse s'exprimer dans un schéma global, voire par un plan territorial détaillé ;

- des stratégies de développement cohérentes entre les différents acteurs ; il s’agit là des principes d'actions, des orientations et priorités, de l'ordonnancement des activités, de l'organisation des processus à mener à moyen et à long terme ;

- des actions concrètes, spécifiques, ciblées, afin de prendre les mesures nécessaires pour mettre en œuvre les stratégies et atteindre les objectifs.

\subsection{La coordination organisatrice}

La multiplication des compétences portées par des entités territoriales aux périmètres non nécessairement concordants suppose la coordination d'un ensemble d'acteurs. Dans le contexte de la coordination entre urbanisme et transports dans les régions urbaines, les conditions d'une telle coordination demandent souvent à être développées et assurées (Kaufmann et al., 2003). Sur le terrain, les compétences et responsabilités morcelées, les intérêts divergents des multiples acteurs compliquent nécessairement la coopération. Des décisions prises sont remises en cause ou retardées. La réussite des projets en la matière reste difficile.

La gouvernance régionale doit prendre en compte les conditions générales et le contexte, que les acteurs régionaux ne peuvent pas changer eux-mêmes, et doit développer des modes de coopération qui répondent au mieux aux problèmes rencontrés. L’examen des différentes expériences menées (Wulfhorst, 2003 et 2006) montre qu'il est utile de commencer la coopération par des projets concrets pour rassembler les acteurs autour d'enjeux partagés et de problématiques communes; élaborer d'abord des pistes de solutions communes, avant d'en déduire les structures organisatrices appropriées. Un projet prometteur trouvera en général le partenariat et aussi le financement nécessaire.

Parvenir à mettre en commun les ressources des différents acteurs et à identifier le bénéfice à attendre pour chacun, sont des conditions nécessaire à la réussite de ces projets. La connaissance et la confiance acquises sur de premiers projets peuvent alors faciliter la délégation de compétences et de budgets, ainsi que la coopération dans d'autres domaines. La coordination organisatrice passera selon les cas par une gouvernance souple à géométrie variable ou par la création d'une autorité commune. Il s'agit alors de déterminer si l'on peut se contenter d'une coopération des acteurs flexible selon les diverses problématiques à traiter ou bien si l’on cherche à intégrer les institutions à un niveau supérieur.

Il est important de préciser que, à la différence de la coopération, l’intégration suppose une réduction de l'autonomie des acteurs en présence. Si donc, sur un territoire donné, on favorise l'intégration pour mieux articuler les politiques d'urbanisme et de transport, on limite ce faisant l'autonomie des acteurs en matière de choix, en particulier celle des collectivités territoriales impliquées (Wiel, 1999), (Kaufmann et al., 2003).

Un enjeu majeur de cohérence consiste à assurer la continuité et la permanence dans ce genre de constellations. Souvent, ce sont les institutions qui, en faisant appel à la mémoire qu'elles ont su engranger, doivent se charger d'éviter les contradictions et de tirer parti des synergies dans le temps 


\subsection{La coordination méthodologique}

D’un point de vue méthodologique, la coordination entre urbanisme et transports nécessite des outils et des processus adaptés. Pour garantir le caractère raisonnable de décisions prises dans un contexte dynamique, il est nécessaire de mettre en place une observation dans le temps de phénomènes fondamentaux touchant à la démographie, l'habitat et les commerces, les déplacements, etc. Alors que cette question est cruciale, en particulier dans un contexte transfrontalier, il manque très souvent une base de données adéquate, qui seule peut fournir les connaissances nécessaires pour établir un diagnostic partagé. Les analyses ultérieures, souvent très poussées, ne peuvent alors empêcher que des décisions de planification ne soient prises sur des bases incertaines, les données incomplètes ne permettant pas de rendre compte d'interactions complexes.

Une mise en œuvre possible de modélisation dynamique consiste à anticiper les effets des décisions et estimer leurs impacts sur le système urbanisme-transports dans une démarche exploratoire. Il s'agit de faire abstraction des interactions réelles entre structures et processus, afin de pouvoir simuler les conditions et effets de différents scénarios de développement. Rappelons à cet effet que tous les modèles sont faux, car procédant toujours par abstraction et représentation incomplète de la réalité, mais que quelques-uns sont utiles, car visant une prise de décision mieux fondée, avec l'ambition d'agir, si ce n'est en connaissance de cause, du moins en connaissance des conséquences.

Pour suivre efficacement la réalisation d'opérations d'aménagement, on peut tester en continu les effets attendus ou les contrôler de façon ponctuelle. Une évaluation des impacts dans le temps vise à s'assurer que le résultat atteint est bien celui qui était attendu et, dans le cas contraire, à apporter éventuellement des mesures correctives On étend ce faisant l'expertise générale sur les interactions dynamiques et enrichit pour le futur la base de connaissances.

Envisager le couple associant ville et transport comme un système nous a permis de mettre l'accent sur la question de la coordination des politiques dans les régions urbaines. Après avoir construit le cadre théorique d'analyse, nous allons maintenant examiner quelques cas d'études, envisagés dans le projet franco-allemand Bahn.Ville 1 pour mettre en évidence les interrelations entre le transport ferroviaire et le développement urbain, avec l'ambition de révéler des pratiques et expériences applicables à d'autres contextes.

\section{Les liens entre offre ferroviaire, mobilité et urbanisme, enseignements du projet Bahn.Ville}

Dans le cadre d'un projet de recherche intitulé Bahn.Ville 1 nous nous sommes attachés à décrire les effets croisés des politiques de développement urbain et de développement des transports ferroviaires régionaux à travers le prisme de trois éléments : la dimension de l'offre ferroviaire, la dimension urbaine et l'espace privilégié de contact entre les deux que sont les gares. Nous présentons ici une série de résultats de cette étude, en mettant l'accent sur les éléments fondamentaux de l'attractivité du transport ferroviaire et le rôle structurant de l'offre ferroviaire, tant du point de vue des pratiques quotidiennes que de celui de la mobilité résidentielle.

\subsection{L'attractivité et la compétitivité du mode ferroviaire}


Les expériences françaises et allemandes de régionalisation de l’offre ferroviaire montrent que celle-ci peut être attractive. Des interviews réalisées dans le cadre du projet Bahn.Ville dans trois des quatre études de cas et ayant impliqué mille usagers et résidants sur chacun des sites il ressort que l'offre ferroviaire régionale est ressentie comme globalement très satisfaisante. La Fig. 2 présente les pourcentages de personnes interviewées qui se déclarent satisfaites ou très satisfaites par l'offre ferroviaire, soit de façon générale (satisfaction globale), soit vis-à-vis des différentes composantes de l’offre de transport qui leur sont suggérées, à savoir la fréquence, la ponctualité, la qualité des correspondances, l'information, les offres tarifaires, la sécurité, la propreté et le confort des trains (moyenne non pondérée). On y voit que l’offre ferroviaire est dans tous les cas appréciée plus favorablement de manière globale que sur des composantes particulières. L’analyse statistique détaillée des liens entre les variables montre par ailleurs qu'on ne peut pas identifier de corrélation significative entre la satisfaction globale et une, deux ou trois des composantes de l'offre. On est alors fondé à poser l'hypothèse que l'offre de transport ferroviaire régional est perçue comme un tout, les différentes composantes agissant en synergie sans que l'on puisse isoler de composants déterminants. Cette hypothèse constitue une invitation à lancer des recherches complémentaires pour mieux comprendre la formation de la satisfaction des usagers face à une offre en transport collectif, dans un contexte où la croissance de clientèle induite par les améliorations de l’offre ferroviaire dépasse souvent les prévisions. Dans la Région Rhône-Alpes par exemple, sur la ligne Grenoble-Rives, la clientèle a augmenté de $170 \%$ entre 1989 et 1993 ; en Alsace, une augmentation de l'offre ferroviaire d'environ $43 \%$ correspond à une augmentation de $50 \%$ de la clientèle et la Bodensee-Oberschwaben-Bahn (ВОВ), dans la région de Ravensburg, dans le Land du Bade-Wurtemberg, est aujourd'hui utilisée par presque quatre mille passagers par jour, soit quatre fois plus que dix ans auparavant.

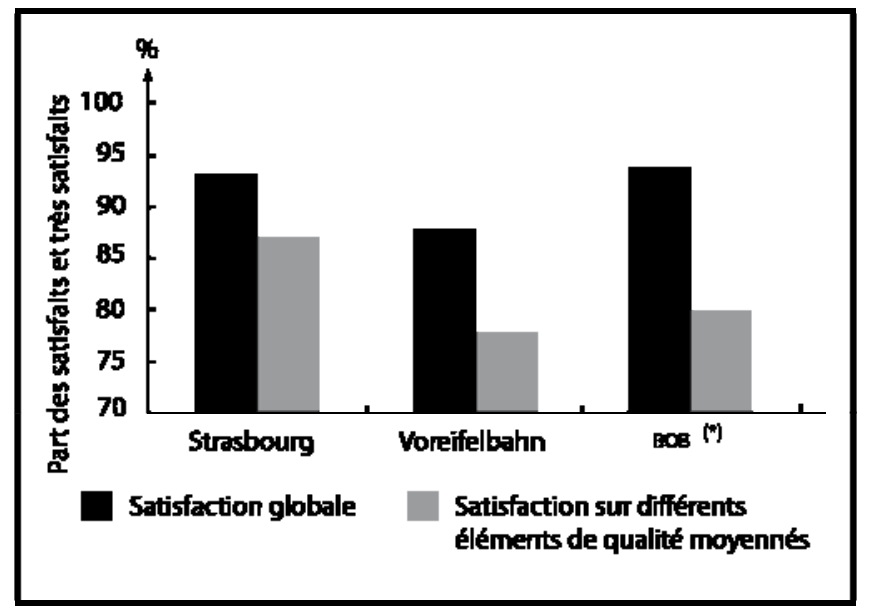

Fig. 2 Appréciation globale de l’offre ferroviaire et appréciation détaillée vis-à-vis de différents éléments de qualité

(*) Bodensee-Oberschwaben-Bahn, ligne de chemin de fer Bodensee-Oberschwaben

Si cette attractivité dépend bien évidemment de la qualité propre de l’offre, elle est aussi tributaire du positionnement de celle-ci par rapport aux autres modes de transport. En effet, au-delà des qualités intrinsèques de l'offre et des facteurs liés à son inscription dans la forme urbaine, l'attractivité du mode ferroviaire dépend des performances des autres modes de transport se trouvant à la disposition des usagers, et plus particulièrement des conditions du déplacement en voiture (situation de trafic/risques de congestion, possibilité de stationnement...). Les raisons du choix du train pour les personnes qui disposent également d'une voiture particulière montrent les facteurs contextuels de succès du mode ferroviaire. À l'échelle d'une région urbaine, le système ferroviaire peut être un mode de déplacement compétitif, surtout grâce à ses qualités de confort, de coût et de performance de desserte. Dans la région 
strasbourgeoise, eu égard aux niveaux de trafic routier enregistrés, on peut poser l'hypothèse que les problèmes liés à la congestion et au stationnement constituent des facteurs essentiels de la relative compétitivité du train, particulièrement pour les déplacements domicile-travail en heure de pointe.

Pour analyser la contribution des systèmes de transport à la structuration des territoires (Stathopoulos, 1997), la mesure de l'accessibilité, comprise comme la plus ou moins grande facilité avec laquelle on peut atteindre un lieu pour y effectuer une activité (Huriot et Perreur, 1994), constitue un moyen privilégié (Rietveld et Bruinsma, 1998), (Haggett, 2001), (Bavoux et al., 2005) (Crozet, 2006). Si l'on admet cette définition, le concept d'accessibilité intègre deux idées différentes et complémentaires (Martellato \& Nijkamp, 1998), à savoir l’idée d'une opportunité de rencontre pour deux agents économiques et l'idée d'une distance à franchir pour réaliser cette interaction. Ces deux dimensions expliquent que la notion d'accessibilité soit partagée entre économie et géographie. De fait, selon qu’est privilégiée la vision économique ou la vision géographique (Bavoux et al., 2005), la mesure de l'accessibilité est caractérisée par un raffinement dans la prise en compte des masses ou un soin porté à la mesure de la distance (L'Hostis et al., 2004), (L'Hostis et Baptiste, 2006).

Tous les éléments recueillis au cours du projet Bahn.Ville tendent à montrer que, globalement, la qualité de l'offre de transport dépend de trois facteurs essentiels, à savoir le confort, le prix du déplacement et les performances de desserte. Toutes ces composantes peuvent être intégrées dans un coût généralisé $\mathrm{c}_{\mathrm{ij}}$ et le paramètre de sa perception $\beta$, qui interviennent dans la formalisation de l’accessibilité (Fig. 3).

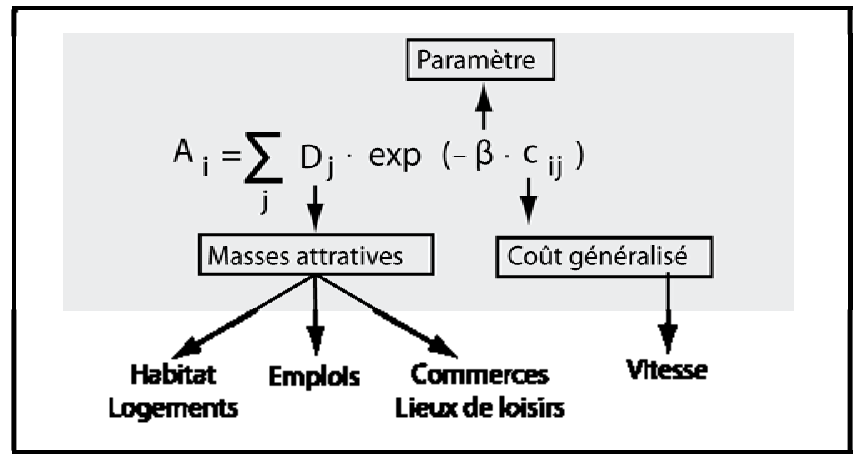

Fig. 3 La formalisation de l’accessibilité par Koenig

D’après (Koenig, 1974)

Pour départager l'offre ferroviaire et l'offre routière en transport individuel, nous reprenons ci-dessous les trois éléments d'appréciation de l'offre ferroviaire, ainsi que les pistes de développement en faveur du transport collectif.

Du point de vue du confort, il est possible de se livrer dans le train à des activités non liées au déplacement comme travailler, lire, se reposer, dormir ou se restaurer, autant d'activités impossibles à mener au volant d'une voiture particulière, mais l'on reste dépendant des horaires. Les pistes de développement concernent dans ce cas l’information et le cadencement de l’offre.

Du point de vue du prix, le système de transport collectif permet ou devrait permettre une articulation des tarifs des différentes compagnies et des abonnements, ce qui suppose une intégration tarifaire, ellemême liée à un partage des recettes. Du côté de la voiture, la structure des coûts, au travers de l'achat, des assurances et des taxes, n’incite pas l'usager à percevoir le lien entre la dépense réelle et la distance parcourue, et au contraire encourage un usage intensif perçu comme permettant une meilleure 
rentabilisation des sommes investies. Les pistes de développement concernent ici la voiture partagée, avec une tarification journalière incluant le carburant.

Du point de vue des vitesses commerciales des différents modes de transport collectif, il faut prendre en compte le temps de parcours global, en incluant les risques de congestion, les temps d'attente et les temps de correspondance. Pour établir une comparaison rigoureuse, cette mesure doit alors être rapportée à celle du déplacement en voiture intégrant les temps de congestion et de stationnement. Le développement du transport collectif peut alors provenir d’une amélioration de la régularité et de la fiabilité de l'offre et d'une augmentation des vitesses de circulation.

\subsection{Un réseau ferroviaire pour structurer les régions urbaines}

Cependant, le mode ferroviaire - moyen de transport relativement lourd en comparaison de la voiture, toujours et partout disponible —, est pertinent pour des flux intenses. La dynamique de l'étalement urbain tend à dégrader cette massification des flux du fait d'une diversification des lieux, des temps et des motifs d’activités. La logique du projet Bahn.Ville indique trois pistes pour répondre à ces enjeux.

- Bahn : adaptation de l’offre ferroviaire grâce à des trains de petite taille et légers, un maillage dense des services et un cadencement (passages assurés aussi en heures creuses, tard le soir et le week-end).

- Ville : réorientation des fonctions urbaines à l'échelle régionale et locale par rapport à un réseau de transports en commun performant, donc renforcement des sites près des nœuds d'accès (gares).

- Le point liant les deux vocables (gares) : accessibilité améliorée par un plus grand nombre de gares (points d'accès au système), des gares plus performantes et accueillantes, voire multifonctionnelles, car assurant des fonctions complémentaires de commerces et de services.

En outre, la cohérence des différentes offres de transport collectif joue un rôle crucial dans l'attractivité du système ferroviaire, car celui-ci peut rarement assurer à lui seul le lien entre le domicile et l'ensemble des activités pratiquées par les usagers. Pour que le mode ferroviaire soit performant, il faut assurer à la fois l'interconnexion physique des infrastructures (accessibilité à pied, quais combinés bustrain, espaces vélos, parcs-relais...) et l'intégration des différents services (tarification commune, horaires adaptés...). Ces enjeux appellent une étroite collaboration entre les acteurs impliqués.

La mise en œuvre du réseau tram-train de l'agglomération de Karlsruhe constitue une illustration de ces principes d'organisation, comme le montre l'évolution du réseau avant et après 1992 en relation avec la forme urbaine. La ligne Karlsruhe-Bretten a été enrichie de quinze nouveaux arrêts pour améliorer la desserte des espaces les plus urbanisés (Fig. 4).

Aujourd'hui, le réseau de tram-train de Karlsruhe continue de s'étendre : d'une longueur totale de $360 \mathrm{~km}$ en 2000 il est passé à $530 \mathrm{~km}$ en 2006 (Fig. 5). Et la fréquentation a fortement augmenté (Fig. 6), avec par exemple une multiplication par huit sur l'axe Karlsruhe-Bretten (histogramme de droite). L'offre ferroviaire constitue un facteur dans les choix individuels de mobilité résidentielle. Le système de tram-train de Karlsruhe est devenu un réseau structurant à l'échelle de la région urbaine. 


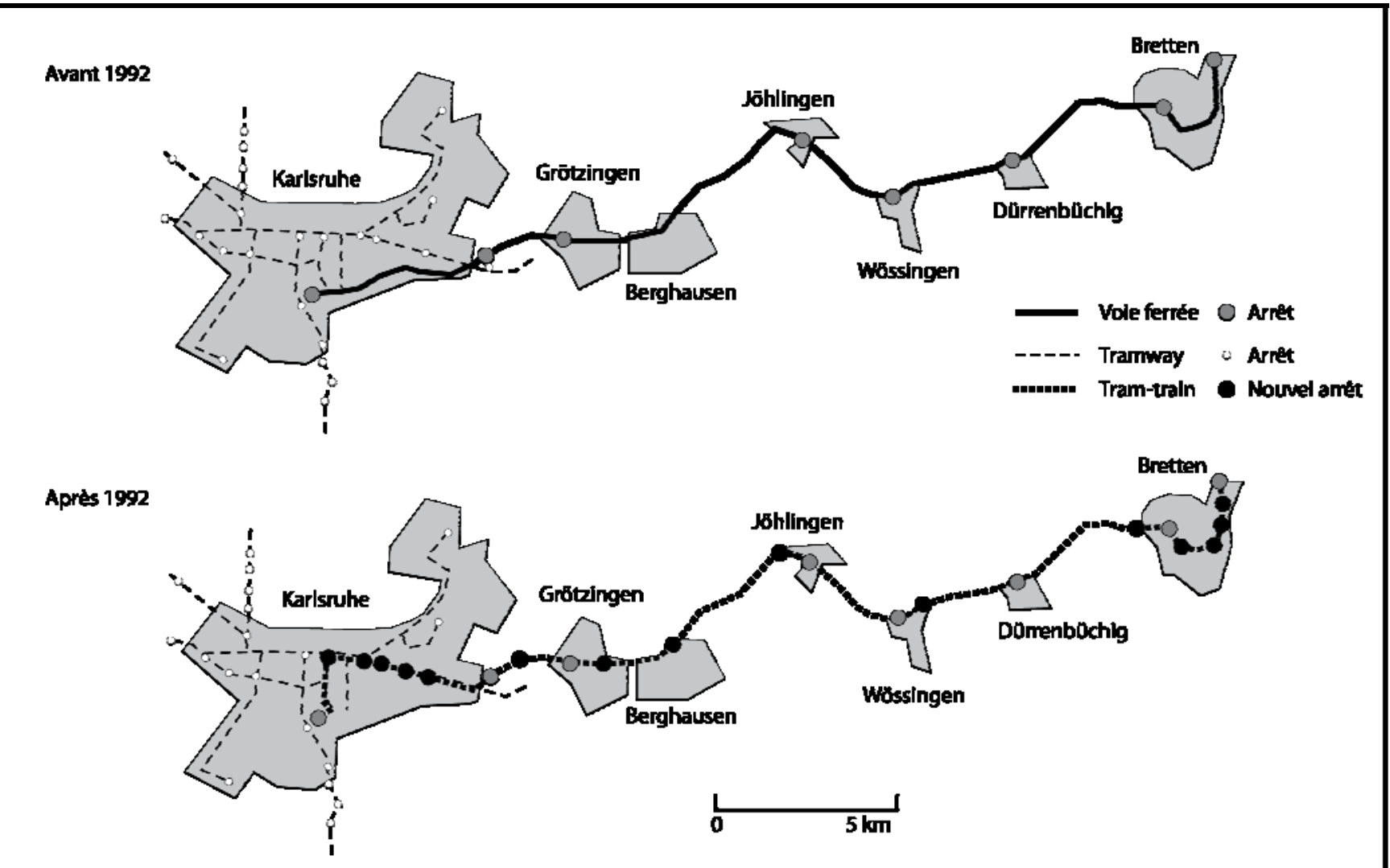

Fig. 4 Évolution du tram-train de Karlsruhe en relation avec la forme urbaine

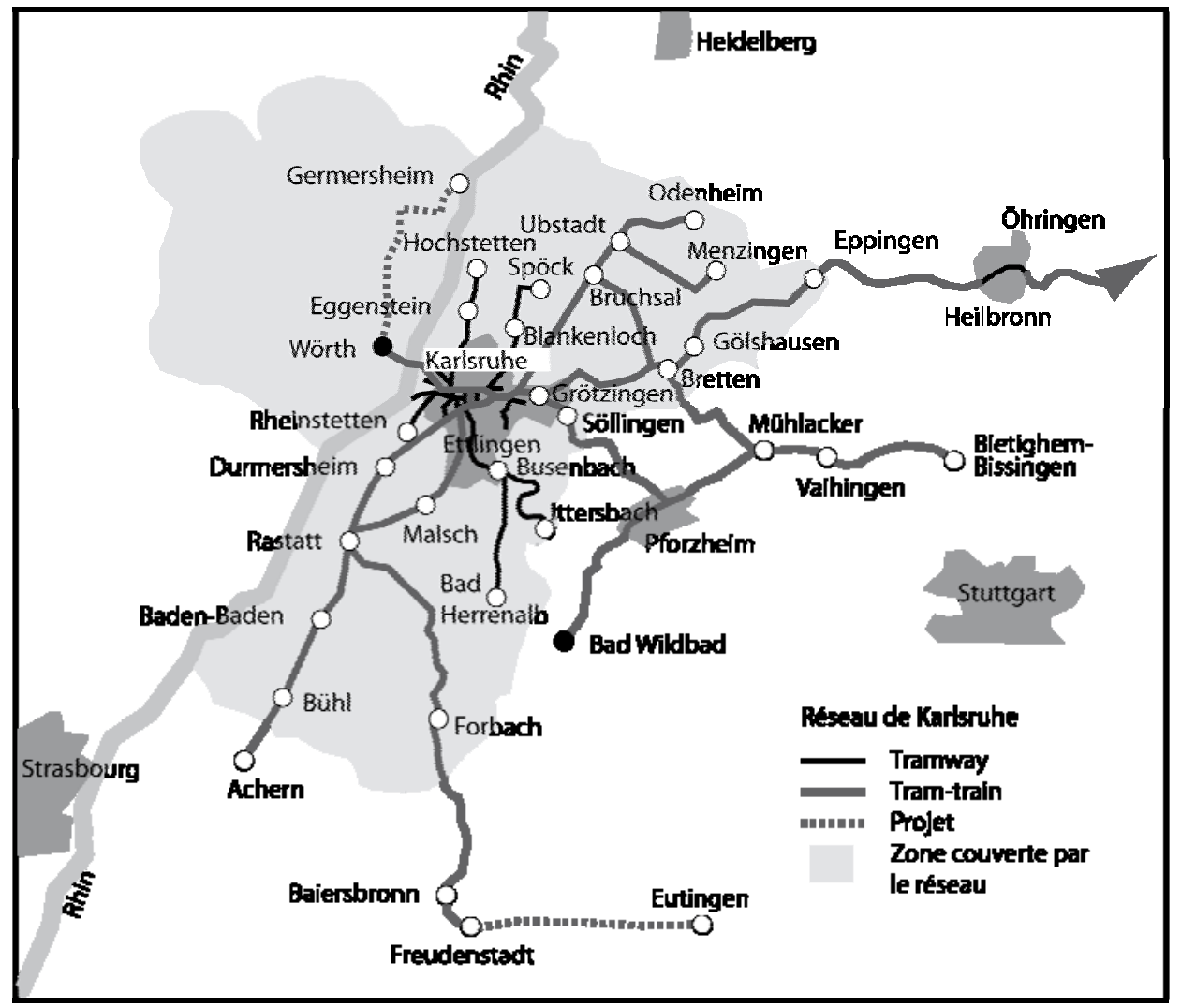

Fig. 5 Le réseau de tram-train de Karlsruhe en 2006 


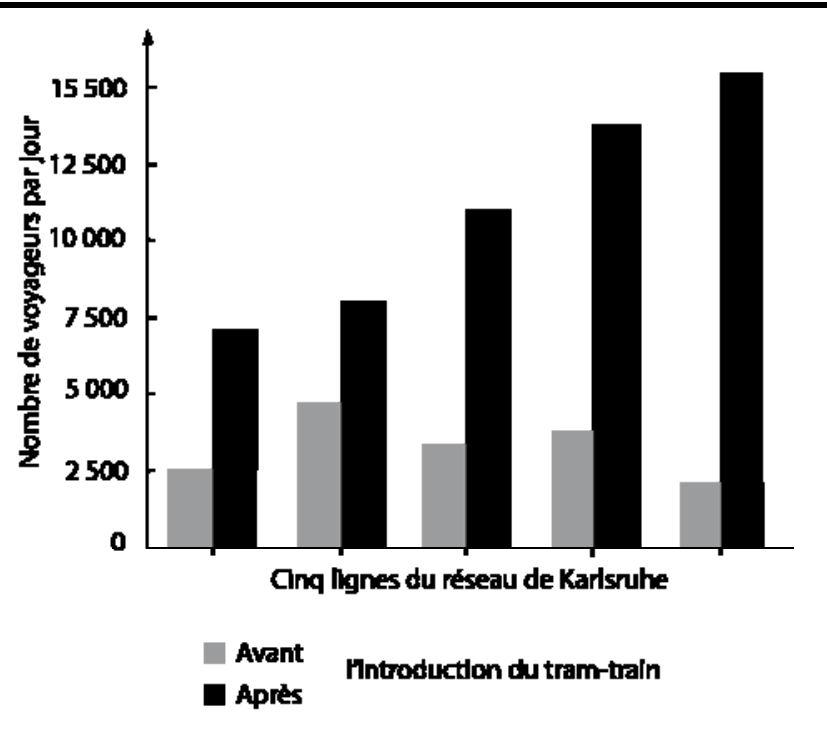

Fig. 6 Évolution de la fréquentation en nombre de voyageurs sur cinq lignes principales du réseau de Karlsruhe avant et après l'introduction du tram-train

Sur le plan historique, la structure urbaine existante a largement modelé la forme du réseau ferroviaire, mais, en retour, ce dernier a aussi contribué à orienter l'urbanisation, et cela dès le milieu du XıXe siècle, avec la création des quartiers de gares. Mais le projet Bahn.Ville n’a pas identifié de lien direct entre l'évolution de l'offre ferroviaire et celle des structures urbaines actuelles, qui englobe les dynamiques spatiales résidentielles et celles des activités, car cette interaction s’inscrit dans un ensemble complexe de décisions individuelles et collectives.

Aujourd'hui, les décisions de mobilité à long terme ne se font pas prioritairement en fonction de la desserte en transport en commun. L'urbanisation se développe plutôt en fonction de ses contraintes propres, des coûts et de l'accessibilité automobile. Sans surprise, la desserte ferroviaire gagne en importance, en tant que facteur de choix dans les décisions de mobilité résidentielle, lorsque les conditions de concurrence entre le ferroviaire et l'automobile sont favorables au train. La comparaison entre le cas strasbourgeois et celui de la вов fait apparaître que la qualité de l'offre ferroviaire est un élément essentiel dans sa capacité à structurer les choix résidentiels. Dans la région BodenseeOberschwaben, l'amélioration de l'offre s'est notamment caractérisée par la mise en place d'un cadencement à la demi-heure et d'une densification des points d'arrêt. Sur la ligne Strasbourg-Saverne, l'accroissement de l'offre, bien qu'important, s'est fait de manière spatialement très inégale. Ainsi, alors qu'entre 1996 et 2004 l'offre s'est accrue de 60 \% sur la liaison Strasbourg-Saverne, dans le même temps et sur la même ligne, la liaison Strasbourg-Dettwiller n'a connu qu'un accroissement de 8 $\%$ du nombre de circulations.

Pour chacune des régions de Strasbourg et BOB, on a recueilli par téléphone l’avis de six cents ménages résidant à moins d'un kilomètre d'une gare. Les déclarations varient certes entre le cas français et le cas allemand, mais, dans les deux pays, les résidants installés depuis moins de trois ans attribuent à la gare plus d’importance que les autres (Fig. 7). Cette évolution dans l'importance accordée à la gare dans les choix de localisation montre que l'offre ferroviaire tend à être un facteur dans les choix individuels de mobilité résidentielle. Pour autant, un développement urbain orienté par le rail est loin d'être spontané. L’enjeu du développement coordonné des transports publics - en particulier du transport ferroviaire — et de l'urbanisme, nécessite la mise en œuvre de stratégies cohérentes entre les différents acteurs des politiques publiques au niveau des régions urbaines. 


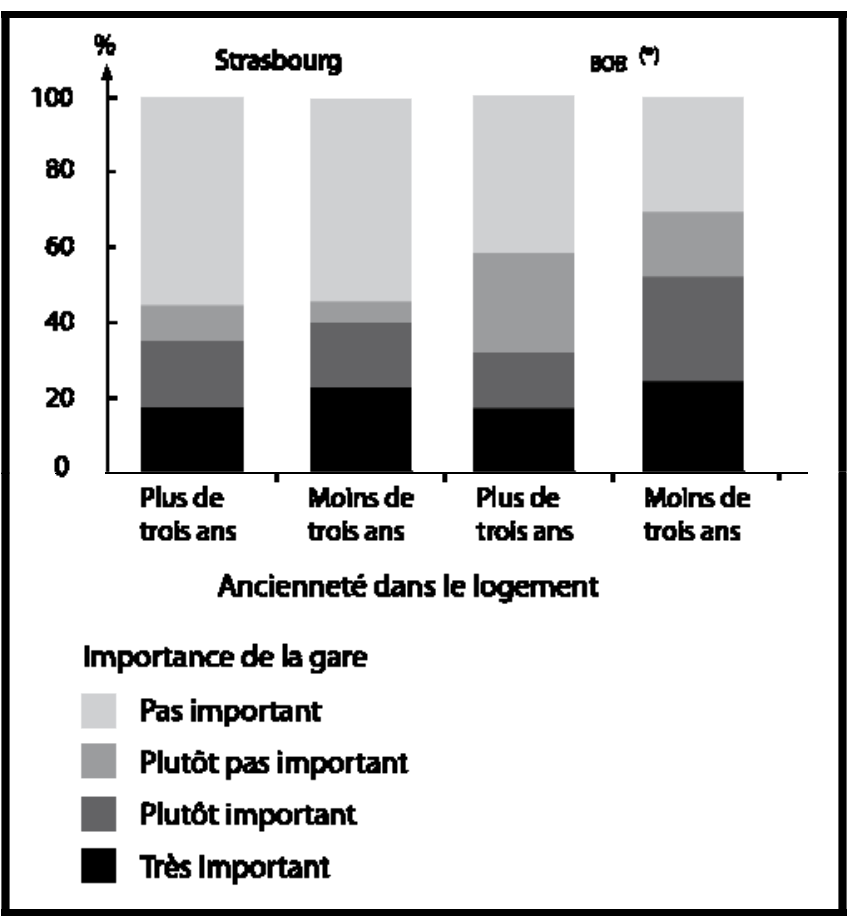

Fig. 7 Importance de la gare dans le choix du logement selon l’ancienneté des résidants

(*) Bodensee-Oberschwaben-Bahn, ligne de chemin de fer Bodensee-Oberschwaben

\section{Perspectives : vers une mise en ouvre}

Nous avons vu au début de l'article que les obstacles à une meilleure articulation entre urbanisme et transport ferroviaire sont nombreux. L’analyse des expériences menées en Allemagne et en France dans le cadre du projet Bahn.Ville 1 a montré les opportunités et les marges de développement à attendre de politiques intégrées fondées sur le mode ferroviaire.

Aujourd'hui se pose avec acuité la question de la mise en œuvre à l'échelle des régions urbaines de ces principes d'un urbanisme orienté vers le rail. À cet effet, un projet de recherche-action intitulé Bahn.Ville 2 démarre en 2007 dans la région de Saint-Étienne, dans le but de mieux mettre en relation le développement de la ville avec celui du mode ferroviaire. Ajoutons qu'une démarche de même ampleur est également prévue du côté allemand, dans la région de Frankfurt am Rhein-Main.

La ligne de chemin de fer Saint-Étienne-Firminy dessert un espace fortement peuplé, dans une ancienne région industrielle confrontée au déclin démographique et à des problèmes de reconversion économique. La Région Rhône-Alpes et la SNCF ont engagé un programme important d’amélioration de la voie ferrée entre Saint-Étienne et Firminy (électrification, fréquence accrue, modernisation des gares) qui s’est concrétisé en 2006. Existe en outre sur cette ligne un grand projet de développement ferroviaire à l'horizon 2010, sous la forme d'un tram-train connecté au réseau urbain de Saint-Étienne. Toutes les collectivités locales ont exprimé un intérêt fort pour le développement de l'intermodalité, qui peut être obtenu par la régénération des quartiers de gare (disponibilités foncières à moyen/long terme liées aux friches industrielles) et par l'amélioration des relations entre les gares, le tissu urbain et les centres historiques. Toutes ces opportunités de régénération urbaine joueraient un rôle significatif dans les perspectives de succès des projets touchant l'offre de transport. C'est pourquoi le site de SaintÉtienne a été choisi pour tester la mise en œuvre d'un urbanisme orienté vers le rail dans le cadre de Bahn.Ville 2. Il s'agit à la fois de mettre au point des outils d'analyse et de simulation, d'aider à la mise 
en œuvre en cours à Saint-Étienne en mobilisant les connaissances accumulées entre autres dans le projet Bahn.Ville 1 et d'identifier les blocages et les facteurs de succès d'une telle orientation.

Ce projet fait l'objet d'une coordination entre de multiples acteurs, parties prenantes des processus qui visent à développer les diverses formes d'articulation entre urbanisme et transport ferroviaire sur un terrain considéré comme un laboratoire en grandeur réelle. Sont à noter l'accent mis sur la question foncière, en relation avec les principes de l'urbanisme des réseaux et le transfert de connaissances de la sphère académique au niveau opérationnel.

Dans le secteur des transports pris isolément, le développement du mode ferroviaire dans les régions urbaines fait intervenir plusieurs niveaux territoriaux, de la Région, autorité organisatrice du réseau régional aux communes accueillant les gares et arrêts, sans oublier les collectivités territoriales métropolitaines, voire les conseils généraux qui ont aujourd’hui compétence pour le développement des réseaux (Zembri, 2004). Cette multiplicité d'acteurs, de compétences et de niveaux territoriaux se retrouve aussi dans les domaines de l'aménagement et de l'urbanisme comme le montre la Fig. 8. S'inscrivant dans une logique de mise en relation d'acteurs multiples, le projet Bahn.Ville 2 doit associer un organisme de recherche (l'INRETS), un pôle d'expertise dans les domaines de l'aménagement et des transports (le CETE de Lyon) et une agence d'urbanisme (EPURES). Les acteurs directement impliqués pour fournir des moyens au projet sont le conseil régional Rhône-Alpes, la communauté d’agglomération de Saint-Étienne-Métropole, et l’organisme public foncier EPORA.

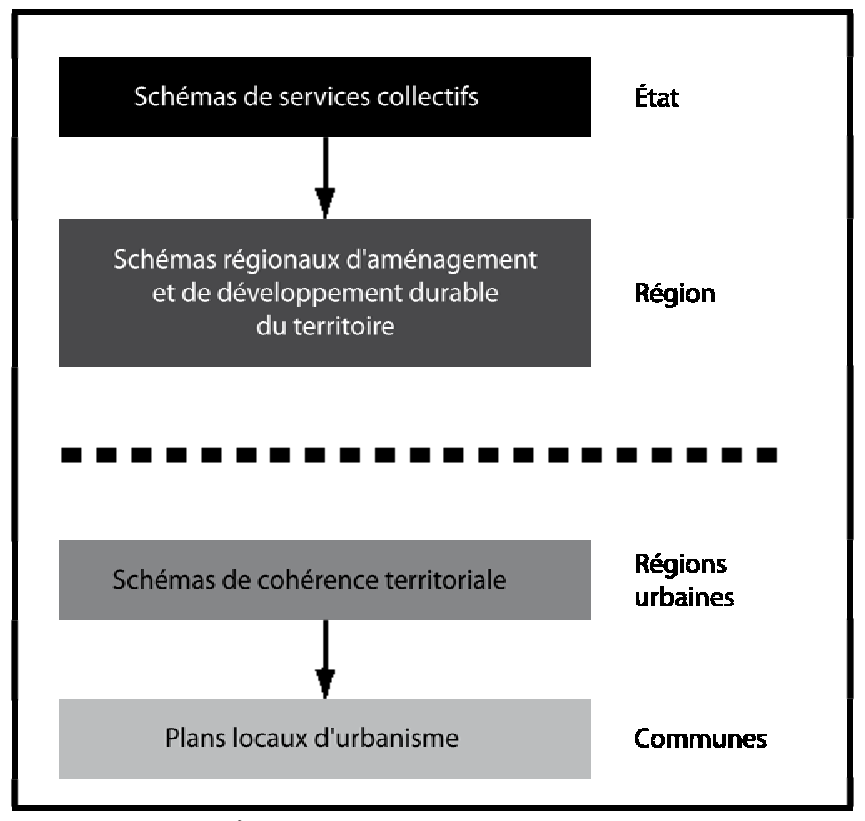

Fig. 8 L'emboîtement des compétences de la planification stratégique à l'urbanisme réglementaire

Dans le développement de la ville, le foncier joue un rôle central. Ainsi, Marc Wiel souligne dans Ville et automobile (Wiel, 2002) le rôle de l'ouverture foncière exceptionnelle occasionnée par la mobilité facilitée et le fait que la croissance urbaine a alors été régulée en facilitant les déplacements plutôt qu'en maîtrisant le foncier. Il appelle alors à la réinvention d'une politique foncière publique devenue quasiment partout évanescente, qui se révèle selon lui d'autant plus stratégique qu'il observe que la meilleure maîtrise de l'éparpillement urbain est le fait d'agglomérations ayant contrôlé le dérapage des coûts fonciers par une offre effectivement disponible et suffisamment abondante en première couronne et donc que, pour maîtriser le degré d'éparpillement de la périurbanisation, il ne faut pas raréfier l'offre 
foncière disponible (par le planification) mais au contraire accroître sa disponibilité réelle en pesant sur les prix là où les collectivités veulent canaliser la périurbanisation. Pour investir les questions foncières sur le terrain de Saint-Étienne, le projet Bahn.Ville 2 prévoit de combiner une analyse des formes urbaines - distribution des fonctions urbaines, typologies d’habitat —, les données issues de la mise en place d'un observatoire foncier continu et pérenne et l'analyse des projets urbains et d'aménagement. Cet examen des dynamiques et des enjeux fonciers sera complété par une observation des pratiques et des conditions de déplacement en mobilisant un outil intermodal d'analyse des temps d'accès, l'analyse spatiale des pratiques de déplacements liés au train et l'étude de la dimension sensible de certains parcours. Elle fournira ainsi sur la mobilité des informations quantitatives et qualitatives susceptibles d'orienter les décisions en matière d'organisation des transports, ainsi que d'aménagement et de développement du territoire. Le croisement de ces deux thématiques, les dynamiques foncières et le fonctionnement des réseaux, a pour ambition de sensibiliser et de familiariser les acteurs locaux à des approches transversales, destinées à susciter la définition de politiques coordonnées et intégrées à même de favoriser un urbanisme orienté vers le rail.

\section{Conclusion}

Si l'évolution de la ville contemporaine doit beaucoup au développement du système automobile, elle ménage aussi des opportunités pour le développement des transports ferroviaires. Envisager le couple ville et transport comme un système permet d'identifier un ensemble de relations causales, ainsi que des facteurs clés susceptibles d'influencer les comportements qui peuvent être actionnés par les acteurs publics. Ainsi l'accessibilité joue-t-elle un rôle charnière en reliant la structuration de l'offre de transport aux choix de mobilité des individus. L'analyse systémique peut aussi être mise à profit pour décliner les figures de la coordination des politiques de transport et des politiques urbaines. Pour parvenir à un développement conjoint des transports ferroviaires et de la ville, nous proposons d'envisager la coordination de secteurs d'intervention sur la ville, la coordination spatiale des échelles urbaines pour dépasser la disjonction des territoires institutionnels et fonctionnels, la coordination temporelle des modalités de l'action et de ses conséquences, la coordination organisatrice au travers des coopérations et de la mise en œuvre de projets communs entre acteurs distincts, et enfin la coordination des méthodes et des outils de suivi et de mesure dans les phases de réflexion et de mise en œuvre.

Le projet Bahn.Ville 1 a été l'occasion d'analyser les différentes dimensions du développement conjoint du rail et de la ville à partir d'une série de cas d'études situés en France et en Allemagne. On a ainsi montré que les réseaux ferroviaires peuvent être porteurs d'une réelle attractivité, à condition de s'inscrire dans une logique d'interconnexion avec les autres modes de transport collectif et la marche à pied et dans une logique d'intégration des différents services liés au transport. À Karlsruhe, le système de transport a vu sa densité de desserte renforcée et son inscription spatiale a été profondément remanié pour mieux répondre au développement de la forme urbaine. L'augmentation très significative de la fréquentation constitue une manifestation de la meilleure adéquation qui a pu être obtenue entre le fonctionnement du système de transport et celui de la ville. Cette attractivité du mode ferré est aussi perceptible au travers des choix de logement tels qu'ils ont été observés par enquête à Strasbourg et à Ravensburg.

La question de la mise en œuvre de ces principes et enseignements sur d'autres espaces est posée au travers du projet de recherche-action Bahn.Ville 2, appliqué sur le terrain de la ligne ferroviaire entre Firminy et Saint-Étienne. À l'échelle d’une agglomération inscrite dans des dynamiques métropolitaines et régionales, le terrain stéphanois constitue un laboratoire privilégié pour étudier et mettre en œuvre les principes d'un urbanisme orienté vers le rail. La forme urbaine héritée d'un passé 
industriel a créé une densité élevée sur le corridor desservi par le réseau ferré, et suscite aujourd’hui l'apparition de vastes espaces mutables, liés aux friches de la désindustrialisation. Si l'on considère que les dynamiques et les décisions publiques en matière de foncier jouent un rôle central dans la production de la ville, ces éléments constituent autant d'opportunités à saisir pour favoriser un urbanisme orienté vers le rail. 


\section{Abridged version}

\section{Introduction}

This paper presents an analysis of the pair formed by the association between a city and rail transport. It will examine the potential for the development of rail networks, the potential for urban development and the interactions between the two.

It is presented in four parts. The first part sets out the general principles of urban organization and the possible directions for urban development policies. We then provide an analysis framework for examining the pair formed by the association between a city and rail transport and identify appropriate levers for action. The third section presents the principal findings of the Bahn.Ville research project which analyzed a series of experiments in France and Germany that relate to rail-oriented urban development. The final section presents some first directions for a future research project that will implement the findings of the Bahn.Ville project on a French site in the Saint-Étienne region.

\section{The principles of urban organization that is in phase with rail networks}

Current thinking about the future of the European city involves forms that are increasingly polycentric and multipolar. Thus, Peter Hall has proposed a reworking of Christaller's central place theory in the light of metropolization (Hall, 1999), in which the European city, shaped by changes in the travel system, is to a high degree structured around the traditional centres and supported by new peripheral centres. Although this urban development is largely determined by the increase in car use (Wiel, 1999), (Mangin, 2004) it creates centres within and between which public transport, particularly rail, can play a major role.

Current changes in cities may provide opportunities for the development of urban public transport networks. However, in order to seize these opportunities the close ties between the city and transport must considered.

\section{The city and transportation as a system}

Many studies have shown that although cities create their own transportation networks they are also to a large degree shaped by them (Dupuy, 1991), (Ragon, 1995), (Beaucire, 1996), (Bretagnolle, 1999), (Menerault and Barré, 2001), (Richards, 2001), (Wachter, 2004), (Menerault et al., 2006). A dialectic exists between the city and transport which must be explored in both directions. While the expansion of transportation networks should be perceived as serving a plan for a city or a region, considering the other direction of the relationship encourages the development of a city that adapts to the constraints and opportunities provided by transport networks.

The system of dynamic links that exists between the city and transportation can be represented as in Fig. 1 (Wulfhorst, 2003). Urban development and the organization of transportation are seen in this context as long-term processes, while travel practices are part of a short-term process. Travel practices are determined by activities and territorial structures which together form transport demand. In particular, daily travel practices use spatial structures to connect the different activity locations. With regard to the long-term processes, accessibility is the link between the urban structure and the transport system. In this context, the future development of urban structures is conditioned by interactions and dynamic processes. 
Analyses of the relationship between urban form and transport accept that the connection between transport policies and planning policies is one of the keys to sustainable urban development (Bertolini et al., 2005), having more importance than either the improvement of public transport or the reduction in the role and use of the car (Laconte, 1999).

While much diagnosis identifies the principal levers, analyses of the experience of European cities ${ }^{2}$ highlight the difficulties of coordinating transportation and planning policies: institutional issues, inconsistency as regards the identification of objectives and confusion between different spatial scales (Jouve, 2003), (Kaufmann et al., 2003), (Schwanen et al., 2004), (Pretsch et al., 2005). The development of rail-based urban projects requires the mobilization and coordination of a large number of players: partnership must play a major role (Klaer Blanchard, 1999), (Puccio and Wulfhorst 2005).

Wulforst (2003) has developed a comprehensive analysis grid in order to reveal the different dimensions of coordination between transport policies and urban planning. The five dimensions of this coordination are set out in Table 1.

Perceiving the city and transportation as a single system brings to the fore the issue of the integration of policies in urban regions. Now that we have constructed the theoretical analysis framework, we shall examine a series of study cases from the Franco-German Bahn.Ville 1 project in order to show the inter-relationships between rail transport and urban development, as well as to identify practices and experience that are applicable to other contexts.

\section{The links between rail transport supply, travel practices and urban development: the lessons from the Bahn.Ville project}

In the framework of a research project known as Bahn.Ville 1 we have attempted to identify the interactions between urban development policies and regional rail development policies with reference to three dimensions: rail transport supply, urban aspects, and the most important interface between the two, the stations. This paper presents a series of findings from this study, paying particular attention to the fundamental aspects of the attractiveness of rail transport, with regard to both daily travel practices and residential mobility.

French and German experience with regard to the regionalization of rail transport supply has shown that rail can be attractive. However, its attractiveness depends both on its intrinsic qualities and its position in relation to other transport modes. Surveys of users and residents conducted in three of the four case studies in the Bahn.Ville project have revealed a very high overall rate of satisfaction with regional rail services (Fig. 3).

However, rail — which is a relatively inflexible transport mode compared to the car which is available at all places and at all times — is appropriate for high level transport flows. The dynamics of urban sprawl tends to work against the concentration of flows as it increases spatial and temporal diversity

2 This paper's focus on European cities should not make us forget other experience, in particular Transit Oriented Development, developed in the United States in the framework of the New Urbanism (Mangin, 2004), (Cervero \& Kochelman, 1997), (CDT, 2002). 
and the range of activities. The Bahn.Ville project aims to respond to these issues by exploring three directions:

- Bahn (Railways): modifying rail transport supply by introducing trains that are small and light, high frequency services, and dense networks (with services that operate in off-peak periods, late in the evening and at weekends, for example).

- Ville (The city): reorganizing urban functions at a regional and local level to be compatible with an efficient public transport network, which will require sites near access nodes (stations) to be improved.

- The full stop between the two words (stations): improving accessibility by increasing the number of stations (points of access to the system), making the stations more efficient and welcoming, and perhaps by providing additional functions (shops and services).

The dynamic revealed by the analysis of the importance of the station in locational decisions (Fig. 8) shows that rail transport supply is starting to become a factor in individual residential mobility decisions. However, rail-oriented urban development is far from endogenous. Coordinating the development of public transport - in particular rail transport - with urban development, therefore requires the implementation of coherent strategies between the different players involved in public policy at urban region level.

\section{Outlook: towards implementation}

Today, the issue of implementing the principles of rail-oriented urban planning at urban region level is posed with urgency. This concern with implementation is the driving force behind the Bahn.Ville 2 research and action project. This project is due to start in 2007 in the Saint-Étienne region and aims to achieve better integration between the development of the city and the development of rail transport.

The Saint-Étienne-Firminy line serves a densely populated area, in an old industrial region faced with demographic decline and problems of economic restructuring. The Rhône-Alpes Region and the French National Railway Company (SNCF) have set out on a major programme to upgrade the railway line between Saint-Étienne and Firminy (electrification, increasing service frequencies, modernizing stations) which is taking concrete shape in 2006. Furthermore, on this line there is also a major rail development project for 2010, a tram-train that connects to the Saint-Étienne urban network. All the local authorities have expressed keen interest in improving intermodality, by regenerating the districts near stations (industrial wasteland which will be available in the medium and long terms) and by improving the connections between stations, the urban fabric and historical centres. These opportunities for urban regeneration could significantly increase the chances of success of projects that target public transport supply. The contextual features underlie the choice of Saint-Étienne as a site for testing the implementation of rail-oriented urban planning, which is the purpose of the Bahn.Ville 2 project. The aim is to develop analysis and simulation tools, to provide assistance to the current implementation in Saint-Étienne by applying the knowledge that has been built up in the framework of Bahn.Ville 1 and elsewhere, and identify the impediments and the factors that favour the success of a policy of this type.

Land use plays a central role in urban development. Thus, in Ville et automobile (2002) Marc Wiel highlighted the role played by the opening up of land use that occurs as a result of easier travel and the fact that urban growth has therefore been regulated by facilitating travel rather than by controlling landHe therefore asks for the reinvention of public policy with regard to land use, which has virtually disappeared almost everywhere. According to Wiel, this is particularly strategic because the 
conurbations that have been most successful in containing urban sprawl are those that have ensured that there is a sufficient amount of available land in the inner suburbs. Thus, in order to limit the extent of suburban growth, it is necessary not to reduce the amount of available land (by planning measures) but on the contrary to increase the amount of land that is effectively available by lowering prices where the local authorities wish to channel suburban development.

To include land use issues in the field in Saint-Étienne, in the context of the Bahn.Ville 2 project it is planned to combine an analysis of urban forms - the distribution of urban functions, the typology of housing - with data obtained from continuous and long-term monitoring of property and the analysis of urban planning projects. This investigation of land use dynamics and issues will be supplemented by observation of travel practices and conditions using a tool that analyzes intermodal access times, spatial analysis of rail travel practices and an investigation of the sensitive nature of some journeys. It will thus provide quantitative and qualitative information about travel practices which may serve to inform decisions with regard to the organization of transport and planning and development in the region. The aim of combining the two issues of land use dynamics and the operation of networks is to make local actors more aware and familiar with cross-cutting approaches which are intended to encourage the development of coordinated and integrated policies capable of encouraging rail-oriented urban development.

\section{Conclusion}

Although the development of contemporary cities owes much to the development of the automobile system, this development also creates opportunities for rail transport. Considering the city/transport pair as a system makes it possible to identify not only a set of causal relationships, but also key factors which can influence behaviours and which may be modified by public actors. Thus, accessibility plays a pivotal role by linking the structure of transport supply to individuals' travel choices. Systems analysis can also be used to explore the various forms taken by the coordination of transport and urban policies. In order to achieve joint development of rail transport and the city, we suggest considering coordination between different sectors of urban action, spatial coordination between the different scales of urban geography in order to overcome the separation between institutional and functional zones, the temporal coordination of action and its consequences, organizational coordination by means of cooperation and the implementation of joint projects involving separate players, and finally the coordination of monitoring and measurement techniques in the study and implementation phases.

The Bahn.Ville 1 project provided the opportunity of analyzing the different dimensions of the joint development of rail and the city on the basis of a series of case studies in France and Germany. It has thus been shown that rail networks can be genuinely attractive, as long as they are interconnected with other public transport modes and walking and that an attempt is made to integrate them with other transport-related services. In Karlsruhe, the density of the transport system has been increased and it has undergone major spatial reorganization to make it more adapted to the development of the urban form. A very significant increase in ridership is the sign of an improvement in the match between the operation of the transportation system and that of the city. The attractiveness of the rail mode is also apparent from the studies of housing choice that have been conducted in Strasbourg and Ravensburg.

The Bahn.Ville 2 research and action programme which is implemented on the Firminy to SaintÉtienne railway line raises the question of applying these principles and lessons in other areas. The Saint-Étienne study area, which covers a conurbation which is experiencing metropolitan and regional dynamics, provides an excellent laboratory for studying and implementing the principles of railoriented urban planning. This area's urban form, inherited from its industrial past, has created high 
population density along the rail corridor and at the present time is leading to the appearance of large areas of modifiable spaces consisting of the wasteland produced by de-industrialization. If one takes the view that public policy dynamics and decisions with regard to property play a central role in the creation of the city, these factors provide as many opportunities for encouraging rail-oriented urban planning. 


\section{Références}

Aguiléra, A., Mignot, D., 2002. Structure des localisations intra-urbaines et mobilité domicile-travail. Recherche Transports Sécurité, 77, 311-325.

Baudelle, G., Peyrony, J., 2005. Le polycentrisme en France : cheminement d'un concept. Territoires, 2030 (1), 89-101.

Bavoux, J.-J., Beaucire, F., Chapelon, Zembri, P., 2005. Géographie des transports. Armand Colin, Paris.

Beaucire, F., 1996. Les transports publics et la ville. Milan Presse, Toulouse.

Bertolini, L., Dijst, M., 2003. Mobility environments and network cities. Journal of Urban Design, 8 (1), 27-43.

Bertolini, L., Le Clercq, F., Kapoen, L., 2005. Sustainable accessibility: a conceptual framework to integrate transport and land use plan-making, Two test-applications in the Netherlands and a reflection on the way forward. Transport Policy, 12 (3), 207-220.

Bretagnolle, A., 1999. Le système de villes dans l'espace-temps : effets de l'accroissement des vitesses de déplacement sur la taille et l'espacement des villes. Géographie, Paris, 320.

CDT, 2002. Transit-Oriented Development Study: Factors for Success in California. California Department of Transportation, 217.

CCE, 1999. Schéma de développement de l'espace communautaire. Office des publications officielles de la Commission de la Communauté européenne, Luxembourg.

Cervero, R., Kockelman, K., 1997. Travel demand and the 3Ds: density, diversity and design. Transportation research, Part D, 2 (3), 199-219.

Crozet, Y., 2006. Du coût généralisé à l'accessibilité : un nouvel horizon pour les politiques de mobilité urbaine ? Intervention au sixième séminaire francophone de socioéconomie des transports.

DATAR, 2000. Aménager la France de 2020 : mettre les territoires en mouvement. La documentation Française, Paris.

Dubois-Taine, G., 2001. La ville émergente : un système de lieux et de liens, réinventer le sens de la ville, les espaces publics à l'heure globale, In : Ghorra-Gobin., C. (ed). L'Harmattan, Paris, 59-66.

Dupuy, G., 1991. L'urbanisme des réseaux. Armand Colin, Paris.

Faludi, A., Waterhout, B., 2002. The Making of the European Spatial Development Perspective, No masterplan. Routledge, Londres, Grande-Bretagne.

Haggett, P., 2001. Geography, a global synthesis. Prentice Hall, Harlow, Grande-Bretagne.

Hall, P., 1999. The Future of cities. Computers, Environment and Urban Systems, 23, 173-185. 
Huriot, J.-M., Perreur, J., 1994. L'accessibilité, In : Auray, J.-P., Bailly, A., Derycke, P.-H., Huriot, J.M. (eds), Encyclopédie d'économie spatiale. Economica, Paris, 55-60.

Jouve, B., 2003. Les politiques de déplacements urbains en Europe, l’innovation en question dans cinq villes européennes. L’Harmattan, Paris.

Kaufmann, V., Sager, F., Ferrari, Y., Joye, D., 2003. Coordonner transports et urbanisme. Presses Polytechniques et Universitaires Romandes, Lausanne, Suisse.

Klaer Blanchard, G., 1999. L'élaboration des PDU et la desserte ferroviaire, Le développement du transport ferroviaire et les plans de déplacements urbains. Presses des Ponts et Chaussées, Paris, 3-18.

Koenig, 1974. Théorie économique de l’accessibilité urbaine. Revue Économique, 275-297.

L'Hostis, A., Menerault, P., Decoupigny, C., 2004. Assessing spatial planning policy with accessibility indicators: the case of Lille's metropolis scenario, In: Beuthe, M., Himanen, V., Reggiani, A., Zamparini, L. (eds), Transport developments and innovations in an evolving world. Springer Verlag, Berlin, Allemagne, 293-312.

L'Hostis, A., Baptiste, H., 2006. A Transport network for a city network in the Nord-Pas-de-Calais region: linking the performance of the public transport service with the perspectives of a monocentric or a polycentric urban system. European Journal of Spatial Development.

Laconte, P., 1999. Villes et transport, l'expérience globale. Transport Public International, 4, 54-57.

Mangin, D., 2004. Infrastructures et formes de la ville contemporaine, la ville franchisée. CERT/Éditions de la Villette, Paris.

Martellato, D., Nijkamp, P., 1998. The Concept of Accessibility Revisited, In: Reggiani, A. (ed), Accessibility, Trade and Locational Behaviour. Ashgate, Aldershot, Grande-Bretagne, 17-40.

Menerault, P., Barré, A., 2001. Gares et quartiers de gares : signes et marges. Rapport INRETS.

Menerault, P., Barré, A., Conesa, A. L'Hostis, A., Pucci, P., Stransky, V., 2006a. Multipolarités urbaines et nouvelles organisations intermodales. Rapport GRRT Nord-Pas-de-Calais, 124.

Menerault, P., Stransky, V., Baptiste, H., Soulas, C., Ollivro, J., Kertudo, C., 2006b. Les pôles d'échanges en France : état des connaissances, enjeux et outils d'analyse. Rapport CERTU, Lyon, France.

Offner, J.-M., 1997. Transport et urbanisme, un régime matrimonial ambigu. Les Cahiers du génie urbain, 13.

Parr, J.B., 2004. The Polycentric Urban Region: A Closer Inspection. Regional Studies, 38 (3), 231240.

Pretsch, H., Spieshöfer, A., Puccio, B., Soulas, C., Leclercq, R., Bentayou, G., 2005. Enseignements du projet Bahn.Ville. Rapport DEUFRAKO. 
Puccio, B., Wulfhorst, G., 2005. Vers un renouvellement des modes de coopération. Dimension villes et territoire, 4-5.

Ragon, M., 1995. L'homme et les villes. Albin Michel, Paris.

Richards, B., 2001. Future transport in cities. Spon Press, Londres, Grande-Bretagne.

Rietveld, P., Bruinsma, F., 1998. Is transport infrastructure effective? Transport infrastructure and accessibility: impacts on the space economy. Springer Verlag, Berlin, Allemagne.

Rouxel, F., Rist, D., 2000. Le développement durable : approche méthodologique dans les diagnostics territoriaux. Rapport CERTU, Lyon, France.

Schwanen, T., Dijst, M., Dieleman, F., 2004. Policies for urban form and their impact on travel: the Netherlands experience. Urban Studies, 41 (3), 579-603.

Stathopoulos, N., 1997. La performance territoriale des réseaux de transport. Presses de l'École Nationale des Ponts et Chaussées, Paris.

Sykes, O., 2005. Polycentricity, An idea that's here to stay? Town and Country Planning, 250-253.

Troin, J.-F., 1995. Rail et aménagement du territoire, des héritages aux nouveaux défis. Édisud, Aix-enProvence, France.

Vodoz, L., 2004. Les mobiles d'une approche territoriale, In : Vodoz, L., Pfister-Giauque, B., Jemelin, C., Les territoires de la mobilité, l'aire du temps. Presses polytechniques et universitaires romandes, Lausanne, Suisse, 1-7.

Wachter, S., 2004. Trafics en ville, l'architecture et l'urbanisme au risque de la mobilité. Éditions Recherche, Paris.

Wiel, M., 1999. La transition urbaine. Mardaga, Sprimont, Belgique.

Wiel, M., 2002. Ville et automobile. Descartes, Paris.

Wulfhorst, G., 2003. Flächennutzung und Verkehrsverknüpfung an Personenbahnhöfen, Wirkungsabschätzung mit systemdynamischen Modellen. Institut für Stadtbauwesen und Stadtverkehr. Rheinisch-Westfälische Technische Hochschule, Aix-la-Chapelle, Allemagne, 310.

Wulfhorst, G., 2006. Intégrer transports et urbanisme au niveau de la région européenne de Strasbourg, Enseignements du projet de recherche TRANSFORMES. Rapport de recherche INSA/ADEUS, www.insastrasbourg.fr/transformes.

Zembri, P., 2004. La planification des transports au niveau régional, Une lecture des évolutions de ces vingt-cinq dernières années. Rapport CERTU, Lyon, France. 